HEART REVIEW

\title{
Percutaneous valve repair and replacement techniques
}

B Munt, J Webb

Heart 2006;92:1369-1372. doi: 10.1136/hrt.2005.067371

Valvular heart disease is a significant cause of morbidity and mortality. Rates increase with age and the prevalence will increase as the population ages. Several factors have led to an interest in expanding percutaneous valve repair and replacement techniques to more lesions in more patients. This review explores current percutaneous valve repair and replacement techniques in mitral stenosis, mitral regurgitation, aortic stenosis and aortic regurgitation in adults, outlines the future directions, and discusses some technique-related issues.

See end of article for authors' affiliations

...........

Correspondence to: Dr Brad Munt, St Paul's Hospital, 2350-1081 Burrard Street, Vancouver, British Columbia, Canada V6Z 1Y6; bmunt@ providencehealth.bc.ca

Accepted 6 November 2005

Published Online First 9 December 2005

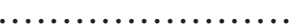

replacement techniques to more lesions in more patients. The role of percutaneous techniques in the treatment of the pulmonary valve has been recently reviewed, ${ }^{7}$ and will not be discussed further. The goal of this review is to explore current percutaneous valve repair and replacement techniques in mitral stenosis, MR, aortic stenosis and AR in adults, to outline the future directions and to explore some issues we believe need to be discussed for the techniques to flourish.

\section{MITRAL STENOSIS}

Percutaneous commissurotomy for native valve mitral stenosis (in valves with commissural fusion) has by far the longest track record and most clinical experience of all percutaneous valve repair techniques. In properly selected patients, clinical success rates (defined as a doubling of mitral valve area from baseline, or increase to at least $1.5 \mathrm{~cm}^{2}$ without production of moderate or severe MR or other significant complications) is 85-99\% depending on patient selection (table 2 ). ${ }^{8}$ Techniques described include single (including the dumbbell-shaped Inoue) and double balloon methods and rigid mechanical devices. The balloons are intended for single use; the prevalence of rheumatic disease in developing countries whose economic profile precludes widespread use of relatively expensive disposable balloons was the impetus for development of the mechanical devices. Multiple randomised trials have shown that percutaneous techniques have equivalent outcomes to surgery in terms of short term results, durability of results and complications. ${ }^{29}$

At our centre, we prefer the Inoue single balloon technique. After the balloon is advanced across the atrial septum (through a transseptal puncture), it is manipulated across the mitral valve. The balloon design allows initial inflation of the distal portion of the balloon that can then be pulled back snug against the ventricular aspect of the mitral valve. Further inflation of the balloon results in inflation of the most proximal chamber that fixes the balloon in position. Lastly, the middle compartment inflates resulting in the augmentation of the valve orifice by commissural splitting. We perform the entire procedure under transoesophageal echocardiography (TOE) and fluoroscopic guidance. Other centres perform the procedure with transthoracic

Abbreviations: AR, aortic regurgitation; EVEREST, Endovascular Valve Edge-to-Edge Repair Study; I-REVIVE, Initial Registry of Endovascular Implantation of Valves in Europe; MR, mitral regurgitation; TOE, transoesophageal echocardiography; $\Pi \mathrm{TE}$, transthoracic echocardiography 
Table 1 Population prevalence of moderate or severe mitral and aortic valve lesions

\begin{tabular}{lll}
\hline Valve lesion & $\begin{array}{l}\text { Prevalence age } \\
\mathbf{6 4 - 7 4} \text { years }\end{array}$ & $\begin{array}{l}\text { Prevalence age } \\
\mathbf{7 5} \text { years }\end{array}$ \\
\hline Mitral regurgitation & 2.8 & 7.1 \\
Aortic stenosis & 1.5 & 4.8 \\
Aortic regurgitation & 0.6 & 1.7 \\
Mitral stenosis & 0.6 & 1.1 \\
\hline \multirow{2}{*}{ Data from Nkomo et al. ${ }^{1}$} & \\
\hline
\end{tabular}

echocardiography (TTE) guidance, but we find that TOE allows continuous imaging without interference with fluoroscopy, and we believe that TOE allows for a safer transseptal puncture than blind or TTE guided techniques. ${ }^{10}{ }^{11}$ TOE also allows echocardiographic monitoring of balloon position as it is advanced into the left ventricle and pulled back. Recently intracardiac echocardiography has been used. ${ }^{12}$ Although imaging of the transseptal puncture and mitral valve may be less optimal with intracardiac echocardiography or TTE than with TOE, endotracheal intubation or a general anaesthetic is not required.

We perform the procedure in the cardiac catheterisation laboratory with the patient under general anaesthesia, intubated and ventilated. The procedure takes about an hour. Patients are typically admitted the day of the procedure and discharged the following morning. The balloon is inflated repeatedly until mild to moderate MR is produced or the maximum balloon diameter is reached.

Percutaneous mitral valvuloplasty is applicable only to patients with valve lesions that involve commissural fusion. As a significant proportion of the mitral stenosis seen in older patients in developed countries results from mitral annular calcification, current techniques will not benefit all of these patients.

\section{MITRAL REGURGITATION}

Given the prevalence of MR and recent observations that even mild MR is associated with an adverse prognosis, ${ }^{13}$ attention has been turned to development of percutaneous techniques to repair the mitral valve. Most percutaneous techniques to treat MR involve repair and fall into one of two general categories: mitral annulus reshaping techniques (including annuloplasty techniques often with the use of the coronary sinus due to its close proximity to the mitral annulus, so called sinoplasty); and methods to effect an edgeto-edge repair after the surgical approach popularised by Alfieri et al. ${ }^{14}$ Whereas valve surgery can have low operative morbidity and mortality in selected patient groups, ${ }^{15}$ comorbidities often associated with ageing, age itself and repeat surgery are independent risk factors for adverse outcomes with surgical valvular procedures. ${ }^{4}$ Traditional valve surgery also usually involves a midline sternotomy with the attendant hospital stay and postoperative pain and recovery time.

All of these factors have led to an interest in expanding percutaneous valve repair and replacement techniques to more lesions in more patients. We characterise the aetiology of MR as structural (gross morphological structural abnormalities of the mitral valve leaflets, chordae and papillary muscles or significant mitral annular calcification) or functional (no structural abnormalities as noted above and left ventricular geometry leading to disruption of the relationships between the mitral apparatus components and a mild degree of mitral annular dilatation). Edge-to-edge techniques are thought to be most useful for prolapse of a middle scallop (A2, P2, or both) or functional MR with a central jet and no severe mitral annular dilatation. Mitral
Table 2 Complications after percutaneous mitral commissurotomy

\begin{tabular}{ll}
\hline Complication & Rate (\%) \\
\hline Mortality & $0-3$ \\
Haemopericardium & $0.5-12$ \\
Embolism & $0.5-5$ \\
Severe mitral regurgitation & $2-10$ \\
Urgent surgery & $<1$ \\
Interatrial shunt & $40-80$ \\
\hline Data from Vahanian et al. &
\end{tabular}

annulus remodelling techniques would be predicted to work best in functional MR where mitral annular dilatation is the dominant abnormality.

Multiple devices for mitral annulus remodelling are under development, including devices by Mitralign, Cardiac Dimensions, MitraLife (ev3), Viacor, QuantumCor and Edwards Lifesciences. ${ }^{16-18}$

The Mitralign ${ }^{16}$ device performs a modified suture annuloplasty by using magnets placed in the coronary sinus and left ventricle to place specialised guidewires in the P1, P2 and P3 zones of the annulus. Specialised anchors are placed at these locations and plicated together to produce a $2-3 \mathrm{~cm}$ reduction in posterior annular circumference.

The Viacor device is based on placing a multilumen delivery catheter into the coronary sinus, and then introducing one or more nitinol treatment devices into the lumens to reshape the annulus. ${ }^{17}$

We have had experience with the Edwards Viking device (Edwards Lifesciences, Irvine, California, USA). This device consists of a proximal and distal self-expanding stent connected by a strut. The strut consists of a spring with an absorbable suture holding the spring in a partially open position. After the coronary sinus diameters and length have been carefully measured before the procedure, the device is placed into the coronary sinus in an introducer. The distal stent is deployed by extruding the device out of the introducer, and it becomes fixed in place by the radial force the stent applies on the coronary sinus wall. The remainder of the device is then deployed, with placement of the proximal stent in the proximal coronary sinus. Over a period of weeks, the suture dissolves, allowing the spring to compress and, because the proximal and distal stents are fixed, the annulus size is reduced leading to a reduction in MR. The approach achieves a $25 \%$ reduction in mitral septal to lateral dimension in animal models. ${ }^{19}$ We have implanted four devices, and MR severity has decreased when the device is intact. A problem has developed, however, with the spring detaching from the proximal stent, and redesign of the device is under way.

The Coapsys device (Myocor Inc, Maple Grove, Minnesota, USA) consists of anterior and posterior pads that are placed on the epicardial surface of the heart connected by a subvalvular cord. The device can be tightened to reduce the left ventricular epicardial to epicardial dimension. Animal models show a reduction in functional MR. ${ }^{20}$

Reported experience in humans with all of the above techniques is small. Immediate success rates, durability of the repair and complication rates are unknown.

The second group of techniques effects an edge-to-edge (Alfieri) repair of the mitral valve. These techniques gain access to the left atrium through a transseptal puncture. The most experience is with the Evalve clip (Evalve Inc, Redwood City, California, USA). Preliminary results of the EVEREST (Endovascular Valve Edge-to-Edge Repair Study) have been presented in abstract form. In 27 patients (out of a planned enrolment of 32) who have received the percutaneous clip, none have died, and the adverse events have been one minor 
stroke and three clip detachments. In all cases, the clip remained attached to one of the valve leaflets and all patients were asymptomatic, the detachment being identified on protocol-driven echocardiography. More than two thirds of the patients achieved a reduction in $\mathrm{MR}$ to $<2+$ at the time of their discharge from hospital. ${ }^{21}$

Retrospective analysis of the cases have indicated that success is more likely when the flail segment prolapses $<10 \mathrm{~mm}$ beyond the mitral annular plane in myxomatous disease and $>2 \mathrm{~mm}$ of mitral leaflet is available for grabbing in functional MR. The randomised EVEREST II trial is underway.

We have had animal and a single human experience with the Edwards Milano II device. This device uses a suction port to capture a mitral valve leaflet and places a stitch through the leaflet. The device is then rotated $180^{\circ}$ and the procedure is repeated. Owing to the needle and suture configuration, once the stitches are placed, as the device is pulled out the needles are exteriorised and a loop of suture is left on the ventricular side of the mitral leaflets, with long tails of both ends of the sutures outside the body. A clip is advanced down the suture tails with a special pusher and tightened up against the mitral leaflets effecting the edge-to-edge repair. Experience in pigs with normal mitral valve leaflets has been promising and has identified the difficulties with current echocardiographic and fluoroscopic techniques to position the device and confirm capture of the leaflets. In our single human experience so far, a patient with isolated $\mathrm{P} 2$ prolapse, we had difficulty positioning the device in the middle of the mitral valve. This resulted in capture of Pl and Al (instead of P2 and A2) and no reduction in the MR severity, so the "bail out" capability of the device was used and no suture was placed.

In our experience, we have identified several issues while performing percutaneous edge-to-edge mitral valve repairs that we believe require solving for the technique to achieve its full potential. Echocardiographic imaging is difficult due to multiple factors including acoustic shadowing off the devices, the tomographic nature of two-dimensional imaging and the need to rapidly confirm the orientation of the devices to the moving mitral valve structures in multiple planes. As well, no standard terminology exists for communicating between the echocardiographer and the interventionalist. For instance, "move the device anteriorly" may mean move the device towards the anterior commissure to some people, towards segment A2 of the anterior mitral valve leaflet to others, and towards the sternum of the patient to yet others. We believe that this terminology problem should be dealt with by international cooperation in developing standard nomenclature and protocols for imaging support of percutaneous valve procedures.

We believe that real-time four-dimensional echocardiography, when it becomes available by the TOE or intracardiac approach, will greatly aid echocardiographic imaging. Device manufactures should work closely with echocardiographers and echocardiographic equipment manufactures throughout the design of their devices to include "echocardiographic friendly" features to allow rapid identification of important landmarks on the devices used in positioning. Lastly, but perhaps most important, it is imperative that physicians involved in all aspects of device development insist on properly conducted clinical trials to assure the safety and efficacy of these devices against current clinical standards of care. Mitral valves of patients with eccentric jets, $\leqslant 3$ segments prolapsing and no severe annular disease (dilatation $>50 \mathrm{~mm}$ or significant mitral annular calcification) can be repaired almost $100 \%$ of the time, ${ }^{22}{ }^{23}$ with very low mortality and morbidity and excellent long-term results (especially for isolated posterior leaflet prolapse).
Percutaneous techniques will need to be well developed before becoming an alternative to surgery for these patients. At the same time, a percutaneous approach for patients who are not candidates for surgery or who choose this approach after being fully informed of the options is well worth pursuing.

\section{AORTIC STENOSIS}

Aortic valve replacement in symptomatic patients with aortic stenosis results in excellent symptom relief and long-term survival in most patients. ${ }^{24}$ Our clinical experience tells us, however, that a growing number of patients are poor surgical candidates due to advanced age, co-morbidities and previous cardiac surgery. Attempts to offer treatment to these patients with balloon aortic valvuloplasty was disappointing, as a durable increase in aortic valve area could not be achieved..$^{25}$ Experience with inflating the balloon across the often heavily calcified stenotic aortic valves did teach us, however, that the balloon could most often be inflated to a significant size. This indicated that the valve could be stretched, but rapid elastic recoil led to the loss of valve area, and few clinically significant embolic events occurred.

Percutaneous aortic valves have been developed, and experience is accumulating in humans. The most experience, including our own, is with modifications of the Cribier valve, now developed by Edwards Lifesciences. Data from the I-REVIVE (Initial Registry of Endovascular Implantation of Valves in Europe) trial in 10 patients show an increase in the aortic valve area and a decrease in the mean transvalvular gradient from preprocedural values of $0.63(0.04) \mathrm{cm}^{2}$ and 45.8 (4.5) $\mathrm{mm} \mathrm{Hg}$ to $1.63(0.07) \mathrm{cm}^{2}$ and $10.5(0.8) \mathrm{mm} \mathrm{Hg}$, respectively, at day $30 .^{26}$

The valve consists of a balloon-expandable stent with equine pericardial leaflets. The valve is available in 23 and $26 \mathrm{~mm}$ diameters requiring insertion through a 22 or 24 French sheath, respectively. Excellent haemodynamic results and durability have been obtained in pulse duplicator simulations.

Initially both antegrade (through a transseptal puncture) and retrograde approaches were being used. Three months after the launch of the first US clinical feasibility trial of the percutaneous aortic valve, however, the antegrade approach was suspended due to the technical complexity of delivering the valve and an unacceptable complication rate. ${ }^{27}$

We use a retrograde approach refined the delivery system. We have not found the same rate of complications. In the retrograde approach the device is delivered directly to the aortic valve, avoiding potential guidewire injury to the mitral valve.

Smaller catheters are used to enable access through the femoral arteries. To get the bulky stent around the aortic arch and through the valve, a special catheter has been developed to steer around the aortic arch and through the aortic valve. So far, we have reported in abstract form retrograde aortic percutaneous valve implantation procedures in 14 patients, of whom 12 were still alive. ${ }^{27}$ All patients were non-surgical candidates. Access site complications are common in a procedure that depends on arterial rather than venous access, and we have observed access site complications in two patients. Femoral access also necessitates removal of the introducer device under direct vision by a surgeon to repair the artery. Postprocedural perivalvular AR is common, but in our experience with a valve stent size correctly matched to the aortic annulus size, the regurgitation will be at most mild. It should be noted that due to the crescent-shaped origin of the AR, quantification by standard echocardiographic techniques can be challenging and regurgitant volume-based approaches measuring stroke volumes at the left ventricular outflow tract and mitral annulus should be used where possible. This appears to be another area in percutaneous 
valve issues where four-dimensional echocardiography may prove useful. Despite these limitations, we believe the retrograde approach will become the procedure of choice for percutaneous aortic valve replacement in the future because it is less demanding technically than the antegrade approach and entails no risk to the mitral valve.

\section{AORTIC REGURGITATION}

Besides the lack of leaflet calcification to guide valve placement, and provided that an adequate size of valve is available, there is no reason that percutaneous aortic valve replacement for AR should differ much from that for aortic stenosis. Animal data indicate that percutaneous aortic valve replacement for $\mathrm{AR}$ is possible.

\section{CONCLUSIONS}

Valvular heart disease is a significant cause of morbidity and mortality. The prevalence of significant (moderate or severe) valve disease in westernised populations is estimated at about $1.8 \%$ of the total population and is increasing as the population ages. ${ }^{1}$ Although traditional valve surgery can have low operative morbidity and mortality in selected patient groups, ${ }^{15}$ co-morbidities often associated with ageing, age itself, and repeat surgery are independent risk factors for adverse outcome with surgical valvular procedures. ${ }^{4}$ These factors have led to an interest in expanding percutaneous valve repair and replacement techniques to more lesions in more patients.

Treatment of mitral stenosis with percutaneous commissurotomy in properly selected patients has outcomes equivalent to traditional surgical procedures and is clinically indicated. ${ }^{2}$

Most techniques to treat MR involve repair and fall into one of two general categories: mitral annulus reshaping techniques, and methods to effect an edge-to-edge repair after the surgical approach popularised by Alfieri et al. ${ }^{14}{ }^{18}$ Preliminary data in humans appear promising with both techniques. In our experience, we have identified several issues while performing percutaneous mitral valve repairs that we believe require solving for the technique to achieve its full potential. Echocardiographic imaging is difficult, and no standard terminology exists for communicating between the echocardiographer and the interventionalist.

Percutaneous aortic valves have been developed and experience is accumulating in humans. The most experience, including our own, is with modifications of the Cribier valve, now being developed by Edwards Lifesciences. Data from the I-REVIVE trial show a good haemodynamic result. ${ }^{26} \mathrm{~A}$ retrograde approach with a refined delivery system seems to be the preferred method of implantation. ${ }^{27}$

Lastly, but perhaps most important, it is imperative that all physicians involved in all aspects of device development and clinical use insist on properly conducted clinical trials to assure the safety and efficacy of percutaneous techniques against current clinical standards of care to produce the best outcome of this promising clinical area.

Since acceptance of this article, the authors' experience with percutaneous mitral annuloplasty ${ }^{28}$ and retrograde percutaneous aortic valve implantation ${ }^{29}$ have been published.

\section{Authors' affiliations \\ B Munt, J Webb, St Paul's Hospital, Vancouver, British Columbia, Canada}

Competing interests: BM and JW are consultants to Edwards Lifesciences LLC.

\section{REFERENCES}

1 Nkomo V, Scott C, Sarano M. Prevalence of valvular heart disease in Olmsted county. J Am Soc Echocardiogr 2005;18:550.

2 Ben FM, Ayari M, Maatouk F, et al. Percutaneous balloon versus surgical closed and open mitral commissurotomy: seven-year follow-up results of a randomized trial. Circulation 1998;97:245-50.

3 Anon. STS Fall 2004 report. Executive summary. http://www.ctsnet.org/file/ STS-ExecutiveSummary-Fall2004.pdf (accessed 21 Sept 2005).

4 Anon. Euroscore calculator. http://www.euroscore.org laccessed 21 Sept 2005)

5 Casula R, Athanasiou T, Foale R. Recent advances in minimal-access cardiac surgery using robotic-enhanced surgical systems. Expert Rev Cardiovasc Ther 2004:2:589-600.

6 Gulbins H, Pritisanac A, Hannekum A. Minimally invasive heart valve surgery: already established in clinical routine? Expert Rev Cardiovasc Ther 2004;2:837-43.

7 Khambadkone S, Bonhoeffer P. Nonsurgical pulmonary valve replacement: why, when, and how? Catheter Cardiovasc Interv 2004;62:401-8.

8 Vahanian A, Palacios I. Percutaneous approaches to valvular disease. Circulation 2004;109:1572-9.

9 Glower D, Landolfo K, Davis R, et al. Comparison of open mitral commissurotomy with mitral valve replacement with or without chordal preservation in patients with mitral stenosis. Circulation 1998;98(19 Suppl):II120-3.

10 Tucker K, Curtis A, Murphy J, et al. Transesophageal echocardiographic guidance of transseptal left heart catheterization during radiofrequency ablation of left-sided accessory pathways in humans. Pacing Clin Electrophysiol 1996;19:272-81.

11 Hahn K, Gal R, Sarnoski J, et al. Transesophageal echocardiographically guided atrial transseptal catheterization in patients with normal-sized atria: incidence of complications. Clin Cardiol 1995;18:217-20.

12 Salem M, Makaryus A, Kort S, et al. Intracardiac echocardiography using the AcuNav ultrasound catheter during percutaneous balloon mitral valvuloplasty. $J$ Am Soc Echocardiogr 2002;15:1533-7.

13 Lamas G, Mitchell G, Flaker G, et al. Clinical significance of mitral regurgitation after acute myocardial infarction. Survival and Ventricular Enlargement Investigators. Circulation 1997;96:827-33.

14 Alfieri O, Maisano F. An effective technique to correct anterior mitral leaflet prolapse. J Card Surg 1999;14:468-70.

15 Moss R, Humphries K, Gao M, et al. Outcome of mitral valve repair or replacement: a comparison by propensity score analysis. Circulation 2003; 108(Suppl 1):1190-7.

16 Baim D. Mitralign retrograde transventricular mitral annuloplasty. In: Valvular Heart Ddisease 2005 AATS Postgraduate Course. Chicago: American Association for Thoracic Surgery, 2005:91.

17 Hayase M. Indirect coronary sinus annuloplasty III. Viacor: animal experimental results, In:Valvular Heart Disease 2005 AATS Postgraduate Course.Chicago:American Association for Thoracic Surgery, 2005:97-8.

18 Alfieri O, Maisano F, Colombo A. Percutaneous mitral valve repair procedures. Eur J Cardiothorac Surg. 2004;26: S36-7; discussion S37-8, (Suppl 1).

19 Kaye D, Byrne M, Alferness C, et al. Feasibility and short-term efficacy of percutaneous mitral annular reduction for the therapy of heart failure-induced mitral regurgitation. Circulation 2003;108:1795-7.

20 Fukamachi $\mathbf{K}$, Inoue $M$, Popovic $Z$, et al. Optimal mitral annular and subvalvular shape change created by the Coapsys device to treat functional mitral regurgitation. ASAIO J 2005;51:17-21.

21 Wood S. Update on percutaneous edge-to-edge mitral valve repair: The EVEREST trial. http://www.theheart.org/ viewArticle.do?primaryKey= 213861 (accessed 21 Sept 2005).

22 Omran A, Woo A, David T, et al. Intraoperative transesophageal echocardiography accurately predicts mitral valve anatomy and suitability for repair. J Am Soc Echocardiogr 2002:15:950-7.

23 Muratori M, Berti M, Doria E, et al. Transesophageal echocardiography as predictor of mitral valve repair. J Heart Valve Dis 2001;10:65-71.

24 Lund O, Magnussen K, Knudsen M, et al. The potential for normal long term survival and morbidity rates after valve replacement for aortic stenosis. $J$ Heart Valve Dis 1996;5:258-67.

25 Otto CM, Mickel MC, Kennedy JW, et al. Three-year outcome after balloon aortic valvuloplasty: insights into prognosis of valvular aortic stenosis. Circulation 1994;89:642-50.

26 Chu J, Cribier A, Baver F, et al. Short-term echocardiographic results in percutaneous transluminal heart valve implantation in patients with critical aortic stenosis: initial registry of endovascular implantation of valves in Europe trial (I-REVIVE) trial. J Am Soc Echocardiogr 2005; 18:543.

27 Wood S. Percutaneous aortic valve trials on hold, pending permission to alter device, technique. http://www.theheart.org/ viewArticle.do?primaryKey $=508017$ (accessed 21 Sept 2005).

28 Webb J, Harnek J, Munt B et al. Percutaneous transvenous mitral annuloplasty: initial human experience with device implantation in the coronary sinus. Circulation 2006;113:851-5.

29 Webb J, Chandavimol M, Thompson C, et al. Percutaneous aortic valve implantation retrograde from the femoral artery. Circulation 2006;113:842-50. 